\title{
La Experiencia de la
}

\section{Reserva Legal en Brasil:}

Una Opción de Conservación en Propiedades Rurales.

Pedro Trejo ${ }^{1}$, Claudia Azevedo-Ramos ${ }^{2}$

\section{RESUMEN}

La Reserva Legal (RL) es un instrumento de protección el cual busca conservar una parte de los recursos naturales en propiedades rurales de Brasil. Desde hace casi un siglo ha sido centro de debates entre productores agropecuarios y ambientalistas. Siendo este un instrumento peculiar de Brasil, a través de una revisión bibliográfica, en este estudio se busca explicar el proceso de evolución de la RL en Brasil con base en su contribución, incluso para la agricultura. Actualmente, parte de los productores apoyan la permanencia de la RL como respuesta a mercados más exigentes con el medio ambiente. Ya habiéndose superado el 100\% del registro de dichas áreas y con un cumplimiento del $80 \%$ la RL empieza a cumplir su función. La mejor comprensión de este instrumento nos permite evaluar su potencialidad para ser utilizada como herramienta para la conservación en diferentes países.

Palabras claves: Brasil, planificación del paisaje, política ambiental, sustentabilidad.

\footnotetext{
1 Doutorando em Desenvolvimento Socio-Ambiental, Núcleo de Altos Estudos Amazônicos (Universidade Federal do Pará). Professor Assistente, Universidad de Los Andes (Mérida, Venezuela), ORCID: https://orcid.org/0000-0002-5700-3072, E-mail: trejopedro21@gmail.com

2 Doutora em Ciências Biológicas, Ecologia (Universidade Estadual de Campinas). Professora Titular, Núcleo de Altos Estudos Amazônicos. Universidade Federal do Pará (Belém, Brasil), ORCID: https://orcid.org/0000-0002-4137-7926, E-mail: claudia.azevedoramos@gmail.com
} 


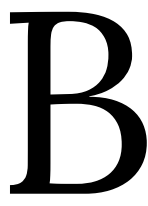

rasil cuenta con un instrumento legal de protección ambiental aplicado en propiedades rurales que, hasta donde sea del conocimiento de los autores, ningún otro país en Latinoamérica y del mundo posee algo similar: la Reserva Legal (RL). Aunque diferentes propuestas han sido elaboradas principalmente en los Estados Unidos ${ }^{3}$, este mecanismo ha ido evolucionando y ajustándose a las realidades de Brasil. En general, la RL es una zona delimitada dentro de la propiedad u ocupación rural, que mantiene las características de este bioma y, por lo tanto, para su aprovechamiento económico se prohíbe la deforestación o la modificación del bioma original (ver detalles más abajo) pero permite el aprovechamiento sostenible. Con su origen en el Código Forestal Brasileño (CF) de 1934 pero con referencias desde su época imperial ${ }^{4}$, la RL obtiene su nombre en el CF de $1965^{5}$ y es reafirmada en el CF vigente $^{6}$. La RL se convierte en un elemento polémico por las diferentes posturas ante esta figura de protección en propiedades rurales a lo largo de su historia.

De manera general, las figuras de protección son restrictivas, es decir, limitan el tipo de actividad e intensidad con que pueden desarrollarse donde son decretadas. Una figura de protección, en Brasil conocida como Unidades de Conservación (UC), puede variar desde la justificación, la intención, la escala, la intensidad, el uso, entre muchas más. Si bien la RL es un instrumento de protección, no es uno tradicional. La diferencia es que las UC se decretan sobre un área pública, por regla general, y es delimitada por un acto del poder público bajo las consideraciones necesarias para el país, estado o municipio. Por su parte, la RL en su totalidad es producto de un único instrumento legal (CF) que la ubica en propiedades rurales limitando su aprovechamiento agrícola y pecuario. Así, se restringen ciertos tipos de actividades en parte de la propiedad rural, pero se permiten otras bajo criterios sociales, ambientales y, por supuesto, económicos. De esta manera, la RL y las UC, son gestionadas de manera diferente.

\footnotetext{
${ }^{3}$ Sergio Ahrens, «Sobre a reserva florestal: origens históricas e fundamentos técnico-conceituais» (Congresso Brasileiro de Direito Ambiental, Meio Ambiente e Acesso à Justiça: Flora, LR e APP, São Paulo, 2007), 691-707, http://www.planetaverde.org/arquivos/biblioteca/ arquivo_20140212142900_2726.pdf.

${ }^{4}$ Daniel Stella Castro, «A instituição da Reserva Legal no Código Florestal Brasileiro: fundamentos histórico-conceituais», Geography Department, University of Sao Paulo, 2013, 132-154, doi:10.7154/RDG.2013.0026.0007.

${ }^{5}$ «Lei n $4.771 / 1965 »$, de 15 de setembro de 1965. Código Florestal. Presidência da República. http://www.planalto.gov.br/ccivil_03/leis/44771.htm.

6 «Lei no 12.651/2012», de 25 de maio de 2012. Código Florestal. Presidência da República. http://www.planalto.gov.br/ccivil_03/_ato20112014/2012/lei//12651.htm.
} 
La aplicación de la RL responde a tres parámetros?: (1) la lógica de la diversificación entre preservación y conservación, (2) la connotación geopolítica y (3) la singularidad territorial, biológica y sociocultural. Brasil es un país que opera en la dualidad preservación-conservación, entendida la primera como la acción de no intervención económica de un espacio y la segunda como el aprovechamiento de los recursos bajo parámetros de sustentabilidad. La RL se ubica en la segunda categoría. La connotación geopolítica está relacionada con los distintos cambios políticos sufridos por Brasil, donde resalta el período de la dictadura militar (1964-1984) en el cual la política de ocupación, integración y control del territorio, más notoria en la Amazonia, llevó a establecer políticas de colonización, pero entendiendo a su vez la importancia de la preservación y conservación de ese ecosistema. Por último, dadas sus características espaciales y de biodiversidad, en las políticas de Brasil, se generaron presiones nacionales e internacionales para aumentar la protección y la variedad de sus áreas. Esto explica, en parte, los diferenciales en los porcentajes de RL según los biomas. En todos estos aspectos, Brasil entendió que la RL era una herramienta que podía cumplir con estos elementos.

Dado el carácter único de la RL en la propiedad rural en Brasil, el propósito de este artículo es aclarar el concepto, las controversias y la situación actual de la RL. Independientemente de la opinión sobre este instrumento, es innegable que la RL de Brasil ha desempeñado un papel relevante en el mantenimiento de la cobertura vegetal y la conectividad entre fragmentos en un país donde el sector del agronegocio corresponde al 21\% del PIB em $2019^{8}$. Adecuadamente adaptado a las particularidades locales, este instrumento puede ser útil para otros países interesados en conservar sus recursos naturales fuera de las áreas protegidas tradicionales.

\section{LA RESERVA LEGAL Y SU POTENCIALIDAD}

\footnotetext{
7 Rodrigo Medeiros y Marta Irving, "A PROTEÇÃO DA NATUREZA NO BRASIL: EVOLUÇÃO E CONFLITOS DE UM MODELO EM CONSTRUÇÃO», RDE-Revista de Desenvolvimento econômico 6, n. 9 (2004): 1-11.

${ }^{8}$ CEPEA, «PIB do agronegócio brasileiro», 2019, www.cepea.esalq.usp.br/br/pib-do-agronegocio-brasileiro.aspx.
} 
La aparición de las primeras ideas para la protección de los bosques en propiedades es de un periodo en que las influencias del "Iluminismo" llegaron a Brasil en el siglo XVIII ${ }^{9}$. Así la transformación de la RL puede considerarse histórica. Apareció oficialmente por primera vez bajo el nombre de Reserva Forestal en el CF de 1934 y fue en el CF de 1965 cuando se le dio el nombre de Reserva Legal.

El actual CF de Brasil decretado bajo la Ley n 12.651 del año 2012, define la Reserva Legal como el "área localizada en el interior de una propiedad u ocupación rural, (...) con la función de asegurar el uso económico de modo sustentable de los recursos naturales del inmueble rural, auxiliar la conservación y la rehabilitación de los procesos ecológicos y promover la conservación de la biodiversidad, bien como abrigo y protección de la fauna silvestre y de la flora nativa." ${ }^{\text {. }}$

De la definición de RL deben entenderse varios elementos claves. Primero, la RL se ubica tanto en una propiedad como en una ocupación rural, así que no se necesita la titularidad para establecer la RL. Por lo tanto, ocupaciones en procesos de otorgación de legalidad deben cumplir con la RL. Segundo, aunque el nombre pueda indicar lo contrario, establece que es posible obtener un beneficio económico de los recursos naturales presentes, por lo que es un área económicamente productiva con base en la oferta que ella misma brinda. Tercero, el uso económico debe respetar los paradigmas de la sustentabilidad. El mismo CF establece el manejo sustentable como la “... administración de la vegetación natural para la obtención de beneficios económicos, sociales y ambientales, respetándose los mecanismos de sustentación del ecosistema objeto del manejo y considerándose, acumulativa o alternativamente, el uso múltiple de especies madereras o no, de múltiples productos de la flora, bien como la utilización de otros bienes y servicios"11.

Un elemento para resaltar es que el aprovechamiento de madera es posible. Además de la función económica de la RL, también debe cumplir funciones tradicionales de las figuras de protección, orientadas hacia procesos ecológicos, biodiversidad y haciendo énfasis en la protección de la fauna y flora nativa. El Estado Brasileño considera posible obtener beneficios económicos en actividades

\footnotetext{
${ }^{9}$ Castro, «A instituição da Reserva Legal no Código Florestal brasileiro», 2013

10 Lei no 12.651/2012. (p. 2).

11 lbid. (p. 3)
} 
sustentables y al mismo tiempo mejorar las condiciones ambientales a través de la RL. Esta situación parece no ser clara entre los detractores de esta figura.

La extensión de la RL dependerá del bioma donde este ubicada la propiedad, siendo la cobertura mínima de 20\% (Cerrado fuera de la Amazonia Legal, Caatinga, Mata Atlántica, Pampa y Pantanal) y la máxima de 80\% (Amazonia y Cerrado en Amazonia Legal). Para propiedades pequeñas en la Amazonia (<20 ha) se exenta la aplicación de la RL. Hay que tener en cuenta que las dimensiones mínimas de una propiedad también difieren entre las regiones de Brasil para que su explotación pueda ser económicamente viable (por ejemplo, 5 ha en el sur y 110 ha en el norte). El porcentaje para la Amazonia puede disminuir para acciones de recuperación de la RL. Si el estado donde se ubica la RL posee Zonificación Económica Ecológica y al menos 65\% de su territorio está cubierto por Unidades de Conservación y Tierras indígenas o si el municipio presenta en mas de 50\% de su territorio Unidades de Conservación y Tierras Indígenas, el porcentaje de recuperación de la RL puede situarse en 50\%. Independientemente del bioma, la RL deberá ser ubicada considerando el plano de la cuenca hidrográfica, la Zonificación Económica Ecológica, formación de corredores ecológicos con otras áreas de importancia ambiental, las áreas de mayor importancia para la conservación de la biodiversidad y las áreas de mayor fragilidad ambiental. ${ }^{12}$

Ya que la ubicación RL es determinada por el propietario u ocupante y considerando el esquema tradicional de producción, es lógico pensar que la RL será ubicada en el área con menor aptitud productiva de acuerdo con las características de la actividad económica que se desarrolla. Así se puede garantizar la máxima productividad. Sin embargo, entendiendo la función de la RL y bajo un esquema productivo no tradicional, se pueden obtener beneficios económicos y conservar los recursos naturales. Mecanismos productivos alternativos como sistemas agroforestales, aprovechamiento de productos forestales no maderables o inclusive turismo pueden brindar un retorno económico importante a partir del manejo de la RL. Si a esta actividad se le suma la posibilidad de que en caso de recuperación del área de la RL se pueden plantar especies exóticas de rápido crecimiento y aprovecharlas en un ciclo único, como mecanismo de aceleración de la sucesión

${ }^{12}$ Lei no $12.651 / 2012$ 
vegetal, los beneficios pueden aumentar significativamente. También existe la opción de mecanismos como los bonos de carbono, es decir, que las opciones para obtener un retorno económico ya existen.

Una figura de protección comúnmente asociada con la RL en Brasil es la Área de Preservación Permanente (APP) (Figura 1). Esta fue creada en el CF de $1934^{13}$ y mantenida en los CF de $1965^{14}$ y de $2012^{15}$. Esta es más restrictiva pues su función está orientada la preservación de cursos de agua y protección ante la erosión u otros procesos degradantes del suelo. Se aplica en el país sin importar si las tierras son públicas o privadas, rurales o urbanas al cumplir con ciertos criterios hidrológicos y/o geomorfológicos.

\section{Figura 1. Representación de la Reserva Legal y Área de Protección Permanente en un conjunto de propiedades rurales.}

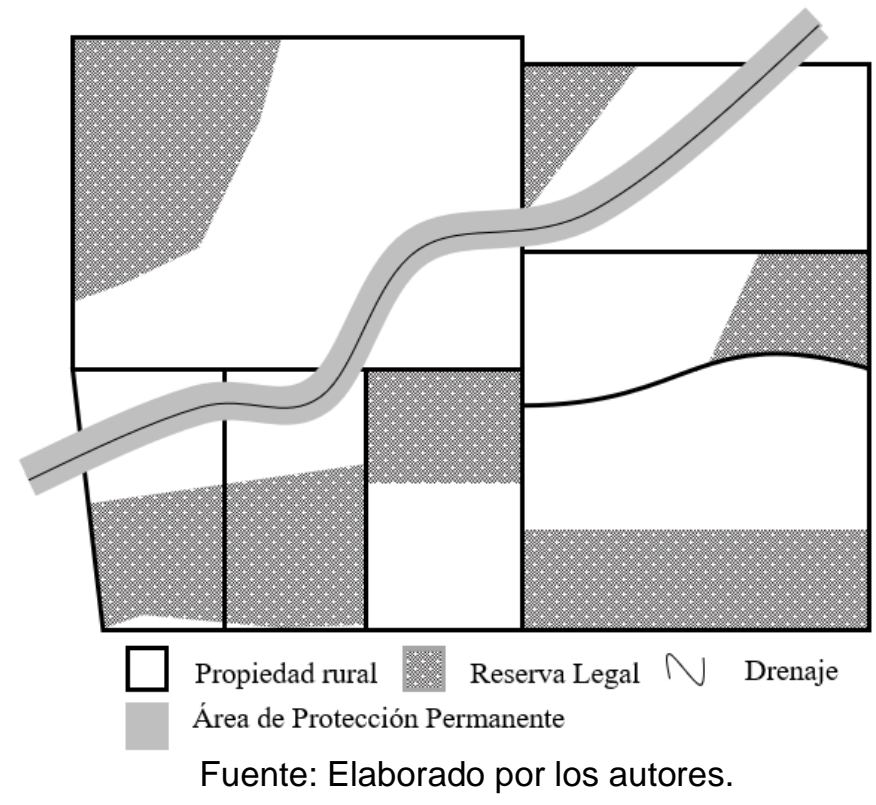

El actual CF brasileño ha establecido un período de dos años a partir de 2012 para que todos los propietarios de tierras registren sus propiedades identificando la RL, APP y otras áreas en un sistema electrónico nacional obligatorio provisto por el gobierno denominado Registro Ambiental Rural (CAR por sus siglas en portugués). El

\footnotetext{
13 Thaís Muniz Ottoni Santiago, José Luiz Pereira de Rezende, y Luís Antônio Coimbra Borges, «A evolução da reserva legal: fundamentos para análise e compreensão do instrumento», Ciência Rural 47, n. ${ }^{\circ}$ (2017), 1-9, doi:10.1590/0103-8478cr20141349.

14 Lei $n^{\circ} 4.771 / 1965$.

${ }^{15}$ Lei no $12.651 / 2012$.
} 
Registro Ambiental Rural o CAR tiene como propósito integrar la información ambiental de las propiedades y es el primer paso para obtener la regularidad ambiental de la propiedad. Aunque este período de registro se ha pospuesto varias veces, de acuerdo con el Comité Técnico del Observatorio del Código Forestal ${ }^{16}$, 96\% de los inmuebles y $80 \%$ de área correspondiente a $3.641 .607,11 \mathrm{~km}^{2}$ cumplen con los requisitos de la RL del CF, es decir, poseen cobertura vegetal nativa o recuperada. Este elemento muestra que ya los productores han sabido adaptarse a esta figura de protección y están dispuestos a cumplir con los parámetros legales que la ley exige, situación diferente en 2010 donde los déficits iban de 1\% (Pantanal) a 34\%

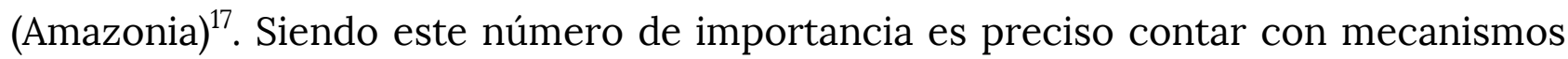
para convertir la RL en áreas económicamente productivas y así reducir su costo de oportunidad para disminuir la presión sobre la vegetación nativa.

\section{CONTROVERSIAS}

La RL ha sido polémica por estar situada en propiedades rurales donde sus beneficios son cuestionados ${ }^{18}$. Siendo esto considerado por algunos actores un aspecto negativo en la dinámica productiva de las áreas, lo que ha ocasionado retrasos en la aplicación de esta, inclusive desde el periodo imperial donde fue establecida una figura primitiva de la $R L^{19}$.

En el CF de 1934 se establecía que el propietario rural al momento de deforestar su propiedad debía resguardar un cuarto de la cobertura forestal nativa. Sin embargo, este instrumento no previó algunos escenarios donde esta área podría fragmentarse de manera virtualmente infinita reduciendo cada vez más el remanente o donde era posible la sustitución de la cobertura nativa por especies exóticas ${ }^{20}$. Esta ley fue de difícil aplicación por el desinterés de conservación de los productores

\footnotetext{
${ }^{16}$ Raoni Rajão et al., «A quem interessa a MP 867 do Código Florestal?» (Observatório do Código Florestal, 2019), 1-14.

17 Gerd Sparovek et al., «Brazilian Agriculture and Environmental Legislation: Status and Future Challenges», Environmental Science \& Technology 44, n. ${ }^{\circ} 16$ (2010): 6046-53.

18 lbid.

${ }^{19}$ Castro, «A instituição da Reserva Legal no Código Florestal brasileiro»; Rodrigo Medeiros y Marta Irving, «A proteção da natureza no Brasil: evolução e conflitos de um modelo em construção», RDE-Revista de Desenvolvimento econômico 6, n. 9 (2004): 11.

${ }^{20}$ Ahrens, «Sobre a reserva florestal: origens históricas e fundamentos técnico-conceituais», 2007.
} 
privados y sobre todo por el poco interés mostrado por el aparato del Estado especialmente a nivel municipal y estadual ${ }^{21}, 22$.

Si bien el CF de 1934 tenía cierto grado de intervencionismo por parte del Estado, este aumentó en el CF de 1965, elemento cónsono con el período de dictadura militar. Sin embargo, esta actualización también representó un avance, especialmente en lo relacionado con la protección y desarrollo forestal. En este se incluyó de manera clara el término de Reserva Legal ${ }^{23}$.

El CF de 1965 sufrió distintas modificaciones ${ }^{24}$. Dentro de estas se encuentran las realizadas por la Medida Provisoria (MP) n².166-67/2001 la cual permitió la creación de la compensación del pasivo ambiental por ausencia de la RL. Siendo posible ubicarla fuera de la propiedad, pero dentro de la misma cuenca hidrográfica, estado y bioma. La reforma también buscaba reducir el tamaño de la RL, situación que no sucedió ${ }^{25}$. Para el año 2004, Brasil tenía más área de protección en RL y APP que en Unidades de Conservación ${ }^{26}$. Esto expresa la importancia de los mecanismos de conservación de la cubierta forestal en el paisaje cuando faltan áreas protegidas.

Debido a la infectividad del CF en proteger áreas naturales rurales y considerado como un elemento que frenaba el desarrollo en la agricultura, se inició en 2010 su revisión ${ }^{27}$, lo que dio origen al CF de 2012, el cual hasta la actualidad se mantiene vigente y presenta características de menor rigidez en las políticas de conservación $^{28}$. Inclusive se encuentra bajo ataque aun cuando para algunos la legislación es demasiado permisiva con los propietarios y, contradictoriamente, para otros es una barrera para el desarrollo ${ }^{29}$. Lo cierto es que el actual CF ya flexibilizó las figuras de APP y RL. Por ejemplo, mientras que el CF anterior exigía que la compensación de la RL fuese en la misma cuenca del mismo estado y en el mismo

\footnotetext{
${ }^{21}$ Sebastião Kengen, «A política florestal Brasileira: uma perspectiva historica» (10 Simpósio Ibero-americano de gestão e economia Florestal, Porto Seguro, Brazil.: Instituto de Pesquisas e estudos Florestais, 2001).

${ }^{22}$ Castro, «A instituição da Reserva Legal no Código Florestal brasileiro», 2013.

23 Sebastião Kengen, «A política florestal Brasileira: uma perspectiva historica» (10 Simpósio Ibero-americano de gestão e economia Florestal, Porto Seguro, Brazil.: Instituto de Pesquisas e estudos Florestais, 2001).

${ }^{24}$ Ahrens, «Sobre a reserva florestal: origens históricas e fundamentos técnico-conceituais», 2007.

${ }^{25}$ Castro, «A instituição da Reserva Legal no Código Florestal brasileiro», 2013.

${ }^{26}$ Medeiros y Irving, «A proteção da natureza no Brasil: evolução e conflitos de um modelo em construção», 2004.

${ }^{27}$ Sparovek et al., «Brazilian Agriculture and Environmental Legislation: Status and Future Challenges», 2010.

28 José Augusto Pádua, «Os Fundamentos Históricos da Conservação Florestal no Brasil», en Ensaios em Ciências Ambientais: Crises, Riscos e Racionalidades. Sandro Dutra e Silva Sayago, D., Toni, F., \& Campos, F. I. (Eds.) (Rio de Janeiro: Garamond, 2016), 185-216.

29 Britaldo Soares-Filho et al., "Cracking Brazil's Forest Code», Science 344, n.o 6182 (25 de abril de 2014): 363-64, https://doi.org/10.1126/science.1246663.
} 
bioma, actualmente sólo se exige que el pasivo ambiental se compense en el mismo bioma, pudiendo variar su ubicación de estado y cuenca (elemento polémico pues puede representar un desbalance en la ubicación de la RL), pero también permite esquemas novedosos de pago por servicios ambientales a través de la Cota Ambiental (déficit de RL de una propiedad compensada por el exceso de otra). En esta situación, sería posible, por ejemplo, que una propiedad en área agrícola extensiva pudiera compensar su RL a varios kilómetros de esta región en un área de menor valor agrícola. Claramente no fueron considerados criterios científicos para estos cambios $^{30}$. Otro cambio importante fue la incorporación de las APP dentro del cómputo de la RL, sim embargo, esto limitaría la producción de actividades sustentables puesto que las APP poseen mayores restricciones que la RL.

El Proyecto de Ley de Conversión $n^{\circ} 9$ (2019) incluye alteración de los requisitos y del proceso que regulan la adecuación de APP y RL en incumplimiento del $\mathrm{CF}$, teniendo efectos negativos en la recuperación de estas áreas ${ }^{31}$. Esta modificación busca establecer como línea base de conservación el estado de vegetación nativa en años anteriores al establecido actualmente por ley, pero sin la obligación de recuperar áreas deforestadas previamente para cumplir con los porcentajes solicitados por el CF. Actualmente, la fecha base para establecer acciones de recuperación es 22 de julio de 2008, es decir, si antes de esta fecha existió desforestación por debajo del porcentaje de la RL, el mismo debe ser recuperado, pudiendo exonerarse en propiedades pequeñas. Desforestaciones después de esa fecha pueden acarrear multas.

Tradicionalmente los debates sobre la modificación de la RL enfrentan a los productores rurales y a los ambientalistas ${ }^{32}$. Los primeros, solicitan la reducción de los porcentajes de área y la flexibilización de los mecanismos de recuperación de las RL por considerar vulnerado su derecho a la propiedad privada y para disponer de estas para la agricultura, puesto que consideran que es mejor incrementar la producción a través del aumento del área productiva. Los segundos, respaldados por la Constitución brasileña, consideran el derecho colectivo a un ambiente sano por

\footnotetext{
${ }^{30}$ Medeiros y Irving, «A proteção da natureza no Brasil: evolução e conflitos de um modelo em construção», 2004.

${ }^{31}$ Raoni Rajão et al., «A quem interessa a MP 867 do Código Florestal?», Observatório do Código Florestal, 2019.

32 Jean P. Metzger, «Bases biológicas para Reserva Legal», Ciência Hoje 31, n. ${ }^{183}$ (junio de 2002): 48-49.
} 
encima del beneficio individual por lo que se debe mantener los porcentajes y defienden que es posible obtener beneficios económicos con el manejo sustentable de esas áreas. Sin embargo, actualmente dentro de los actores que defienden la RL se encuentra parte del sector del agronegocio ya que consideran que el posicionamiento de los productos en los mercados internacionales puede verse perjudicado si son producidos bajo parámetros que afectan aún más al medio ambiente ${ }^{33}$.

Aunque la justificación para cualquier cambio relacionado con la RL es económica, la razón para tomar la decisión es política, pero esta debería considerar aspectos ecológicos ${ }^{34}$ como los servicios ecosistémicos ofrecidos por los procesos ecosistémicos que ocurren en la RL.

Un elemento para también tomar en cuenta es que no necesariamente más deforestación significa mayor desarrollo. La Amazonia brasilera no necesita deforestar más para aumentar las ganancias. Por un lado, se ha demostrado que el aumento de la deforestación no incide necesariamente en el mejoramiento de la calidad de vida de los pobladores de las áreas afectadas y por el otro, con la tecnificación de los procesos productivos ya establecidos se podría aumentar las ganancias, siendo innecesaria la deforestación en nuevas áreas para aumentar el beneficio económico ${ }^{35}$.

Una vez instituido el CF de 2012, los propietarios rurales tuvieron que adecuarse a las normas. Aunque los ambientalistas consideran que hubo retrocesos legales, la adhesión del 100\% de las propiedades en la delimitación de sus RL seguramente trajo beneficios, como la conservación del agua, el suelo, la cobertura forestal y la conectividad entre fragmentos forestales, beneficios que deberían aumentar con la próxima etapa denominada Programa de Regularización Ambiental. Con los nuevos sistemas de regularización ambiental de las propiedades implementadas por el gobierno brasileño (por ejemplo, Registro Ambiental Rural), se aumenta la transparencia de la información y la gobernanza ambiental. Las agencias

\footnotetext{
${ }^{33}$ Coalizão Brazil, «Em defesa do Código Florestal», 2019, http://www.coalizaobr.com.br/home/emdefesadocodigoflorestal.

${ }^{34}$ Metzger, «Bases biológicas para Reserva Legal», 2002.

35 Paulo Moutinho et al., "A Pathway to Zero Deforestation in the Brazilian Amazon Full Report», 2017, 1-33; Sociedade Brasileira para o Progresso Da Ciência y Academia Brasileira de Ciências, O Código Florestal e a Ciência: contribuições para o diálogo, SBPC, 2012, 1-149. Marcelo C. C. Stabile et al., "Solving Brazil's Land Use Puzzle: Increasing Production and Slowing Amazon Deforestation», Land Use Policy, 19 de noviembre de 2019.
} 
ambientales pueden usar el sistema como un aliado para la gestión y planificación del paisaje y la sociedad tiene una herramienta relevante para el monitoreo.

Si bien el registro de la RL en las propiedades en un sistema informático permite el monitoreo y control a su vez también limita la adhesión de los propietarios que realizan actividades ilegales. Y cuando ocurren ilegalidades el Estado posee limitaciones para actuar. Para forzar a los ejecutores de actividades ilegales el Estado puede ofrecer incentivos o aplicar sanciones. En el caso de la RL, donde hay un alto nivel de impunidad para sanciones ambientales, no parece haberse tampoco creado los incentivos necesarios en los productores, especialmente en la reducción de la deforestación o en la recuperación de las áreas ${ }^{36}$. De esta manera se identifica que al momento de que algún país desee aplicar un mecanismo parecido debe considerar los incentivos necesarios para que la medida tenga éxito, puesto por las características de las áreas rurales donde la escasez de servicios limita las acciones del Estado, mecanismos alternativos deben ser implementados.

Una situación particular se presentó con este mecanismo en el Estado de Pará. Al evaluar el desempeño ambiental de los municipios a partir de la descentralización y por seis años (2009-2015) el CAR tuvo un impacto negativo en el índice de desempeño ambiental $^{37}$. Es decir, el CAR empeoró, en un principio, el desempeño ambiental. Esto debido a que los ocupantes ilegales de lotes de terrenos empezaron a deforestar áreas y dejar con cubierta vegetal identificándolas como RL para proceder a registrarlas esperando que ese proceso les otorgara legalidad sobre la tierra. Posteriormente el CAR pasaría a influir de manera positiva en el desempeño ambiental del municipio. Esto demuestra la complejidad de una política ambiental y las necesidades de monitoreo en su implementación.

Aun así, con los inconvenientes mencionados durante el periodo 2006-2013 la deforestación se redujo en un 10\% en los estados de Pará y Mato Grosso gracias a la aplicación del registro de las propiedades rurales en el CAR ${ }^{38}$. Al considerar la RL y APP que el CF dicta proteger se está hablando de 2.810 .000 de $\mathrm{km}^{2}$ una superficie

\footnotetext{
${ }^{36}$ Andrea A. Azevedo et al., "Limits of Brazil's Forest Code as a means to end illegal deforestation», Proceedings of the National Academy of Sciences 114, n. ${ }^{\circ} 29$ (18 de julio de 2017): 7653-58, doi:10.1073/pnas.1604768114.

${ }^{37}$ Benedito Silva, Claudia Azevedo-Ramos, y Hilder Farias, «The impact of decentralization policies: the environmental performance applied to municipalities of the Amazon», Desenvolvimento e Meio Ambiente 51 (agosto de 2019): 202-23.

${ }^{38}$ Moutinho et al., «A Pathway to Zero Deforestation in the Brazilian Amazon Full Report», 2017.
} 
considerable que debería estar cubierta de bosque nativo ${ }^{39}$ brindado múltiples servicios ecosistémicos, elemento clave considerando la afectación que puede sufrir la Amazonia debido al Cambio Climático ${ }^{40}$.

\section{CONTEXTO ACTUAL Y PROSPECTIVA DE LA RL}

Las disputas sobre RL, sus propósitos y necesidades de recuperación, en cierto modo, se pacificaron después que el nuevo CF 2012 fue promulgado. Los ganadores o perdedores se centraron más en el despliegue del CF. Sin embargo, con las elecciones presidenciales de 2018, un gobierno de pocas preocupaciones ambientales y con muchos votantes en el sector agrícola, se comenzó a fomentar nuevas disputas. Recientemente (2019), un proyecto de ley (PL 1551/2019) que proponía eliminar la RL, de autoría del senador e hijo del presidente de la república, fue presentado al Congreso Nacional. Sin embargo, los congresistas juzgaron que el desgaste con la sociedad brasileña tendría un alto costo y retiraron la propuesta de la pauta de votación.

Según el Servicio Forestal de Brasil, que es el administrador del Registro Ambiental Rural, para julio de 2019, se registraron 6.1 millones de propiedades rurales, con un área total de 5.276.070,95 $\mathrm{km}^{241}$. Esto es un $32 \%$ más que el área registrable (3.978.368,64 $\left.\mathrm{km}^{2}\right)$. Aunque parezca un error del sistema, lo que indica son disputas de tierras en un complejo sistema de tenencia de la tierra. Exponer esta situación es otro beneficio del Registro Ambiental Rural, pero está fuera del alcance de este estudio.

El Programa de Regularización Ambiental (PRA) es un proceso bajo la responsabilidad de los estados (o provincias) donde se busca, entre otros, identificar y mejorar la recuperación de la RL. Como para esto es necesario resolver los conflictos de propiedad, existe una gran inquietud de como este proceso podría llevarse a cabo. Lo cierto es que en esta fase es cuando se diseñarán las estrategias para dar

\footnotetext{
${ }^{39}$ Andrea A. Azevedo, Marcelo C. C. Stabile, y Tiago N. P. Reis, «Commodity Production in Brazil: Combining Zero Deforestation and Zero Illegality», Elementa: Science of the Anthropocene 3 (2 de diciembre de 2015): 1-12, doi:10.12952/journal.elementa.000076.

40 Philip B. Duffy et al., «Projections of Future Meteorological Drought and Wet Periods in the Amazon», Proceedings of the National Academy of Sciences 112, n. 43 (27 de octubre de 2015): 13172-77, doi:10.1073/pnas.1421010112.

41 SFB, «Cadastro Ambiental Rural Boletim Informativo», Datos hasta 31 de julio de 2019, 2019, http://www.florestal.gov.br/documentos/car/boletim-do-car/4211-boletim-informativo-julho-de-2019/file.
} 
cumplimiento a lo establecido en el CF por aquellos que se encuentren fuera de la norma.

Se puede decir que el escenario a futuro ideal de la RL sería aquel en el cual las propiedades rurales están cumpliendo con los porcentajes de cobertura (o que ya ubicada la RL está en proceso de recuperación); posean una cobertura natural o antrópica que se asemeja a la original principalmente en servicios ecosistémicos; están conectadas entre ellas mismas y/o con otras áreas de interés ambiental y son productivas económicamente bajo criterios de sustentabilidad. De cumplirse estos elementos podríamos decir que este mecanismo está funcionando en su totalidad. Como ya se ha explicado anteriormente, la situación actual está lejos de ese escenario ideal planteado, sin embargo, todas las acciones que se han ejecutado y la perseverancia de la sociedad civil brasileña apuntan al cumplimiento del marco legal a pesar de los obstáculos que han estado presentes.

\section{AMÉRICA LATINA Y LA RESERVA LEGAL}

El instrumento RL puede tener una aplicación práctica en otros países, especialmente en aquellos con pocas áreas públicas o áreas protegidas. A continuación, analizaremos, como ejemplo, su aplicación en América Latina y el Caribe, dadas las similitudes geopolíticas y sociales.

América Latina y el Caribe tienen 23,22\% y 13,1\% de área terrestre y marina, respectivamente, bajo alguna figura de protección ${ }^{42}$. Aun así, en la actualidad existe a nivel regional una preocupación por el mantenimiento de estas áreas de protección. Procesos como Degradación, Reducción y Desincorporación de Áreas Protegidas, conocido como PADDD (por sus siglas en inglés) ${ }^{43}$, son cada vez más presentes. Hasta 2018, se registraron 544 eventos afectando un área de $630.197 \mathrm{~km}^{2}$ en América Latina y el Caribe ${ }^{44}$. Estas acciones debilitan el sistema de áreas protegidas de los países, con

\footnotetext{
42 UNEP-WCMC, «Protected Area Profile for Latin America \& Caribbean from the World Database of Protected Areas, September 2019», 2019, www.protectedplanet.net.

43 Michael B. Mascia y Sharon Pailler, «Protected Area Downgrading, Downsizing, and Degazettement (PADDD) and Its Conservation Implications: PADDD and Its Implications», Conservation Letters 4, n. ${ }^{\circ} 1$ (febrero de 2011): 9-20, doi:10.1111/j.1755-263X.2010.00147.X.

44 Conservation International y World Wildlife Fund, «PADDDtracker.org Data Release Version 2.0 (May 2019). Arlington, VA: Conservation
} 
impactos directos en los procesos ecosistémicos que son de las principales razones para su creación. Acciones como la construcción de infraestructura y minería son las principales causas para la afectación de las áreas protegidas en la región ${ }^{45}$. Para hacer frente a esto, la sociedad civil se ha organizado y con apoyo de fondos internacionales o propios han implementado la creación de áreas de protección privadas a través de la obtención áreas para transformarlas en reservas privadas ${ }^{46}$. En este tipo de iniciativa México, Chile y Perú son los países que presentan mayores áreas registradas ${ }^{47}$.

Sin embargo, las iniciativas de crear áreas privadas de conservación no garantizan la conectividad entre ellas o con otras áreas de interés ambiental, creando la necesidad de crear corredores ecológicos. Así se haría necesario también establecer mecanismos para conectar estas áreas a través de los diferentes tipos de propiedad o uso, elemento que la RL puede resolver. Estos mecanismos deben ser aplicados por la autoridad ambiental. Esta autoridad en principio es la encargada de aprobar la ubicación de la RL previa propuesta del propietario. En caso de no satisfacer los requerimientos de conectividad la autoridad puede proponer una nueva ubicación y se iniciaría un proceso de negociación.

Teniendo América Latina una alta presencia de propiedades rurales, se puede establecer mecanismos alternativos o de apoyo a la creación de áreas protegidas para proteger la vegetación nativa. Para que la RL tenga éxito como estrategia de conservación sería ideal que no existan conflictos con la propiedad de la tierra, sin embargo, mecanismos como la RL pueden evidenciar donde están presentes estos problemas, como está ocurriendo actualmente en Brasil.

También es necesario un marco legal claro que no entre en conflicto con otras leyes (especialmente ambientales) y donde se estipule las características que debe poseer el área destinada a ese uso, el porcentaje de área a conservar, el uso permitido, la ubicación, entre otras.

\footnotetext{
International. Washington, DC: World Wildlife Fund.», 2019.

45 Ibid.

${ }^{46}$ Claudio Rivera y Arturo Vallejos-Romero, «La privatización de la conservación en Chile: repensando la gobernanza ambiental», Bosque (Valdivia) 36, n. ${ }^{\circ} 1$ (2015): 15-25, doi:10.4067/S0717-92002015000100003.

${ }^{47}$ UNEP-WCMC y IUCN, Protected Planet Report 2016 (UNEP-WCMC and IUCN: Cambridge UK and Gland, Switzerland., 2016).
} 
Un sistema de monitoreo y control es imperativo. Brasil está implementando el SICAR, esta plataforma permite que cada propietario registre de manera individual su propiedad. Herramientas de este tipo son útiles siempre y cuando no se presten para la apropiación ilegal de la tierra y su manejo sea eficaz. Si bien el mecanismo anterior es solo de registro también se hace necesario crear mecanismos para monitorear el cumplimiento del marco legal en la titularidad de las tierras.

Otro elemento de gran importancia es la creación de incentivos para que los propietarios acepten la RL, más allá de servicios por pagos ambientales o permitir actividades productivas sustentables (elementos que también deben existir), es necesario que la implementación de la RL no genere una pérdida económica significativa al propietario y si es así deben existir los elementos que compensen esa pérdida, es decir, respeto a la producción económica a través de diferentes alternativas de producción y estimular la creación de mercados de productos provenientes de estas áreas y cualquier otro elemento necesario que se ajuste a la realidad del país o región que desee incorporar estos mecanismos.

También disminuirían la presión en grandes áreas para ser decretadas figuras de protección y a su vez distribuirían la responsabilidad de la conservación a la sociedad (sin que esto signifique que las áreas públicas no deben ser protegidas, sin duda alguna existen áreas públicas donde es necesaria la máxima restricción de uso). Los propietarios asumirían responsabilidad directa por la conservación y la sociedad se beneficiaría por los servicios ecosistémicos brindados por estas áreas (en particular la conservación del agua para consumo humano), reconociéndolo debidamente a los propietarios. Así se estarían ejerciendo acciones locales con impactos principalmente locales y, por supuesto, globales. Por lo tanto, mecanismos como la RL, pueden ser de gran ayuda pues reparten la responsabilidad en la sociedad que a final de cuentas son los usuarios de los servicios ecosistémicos que estas áreas proveen y reafirmándoles el derecho a exigir políticas ambientales al Estado.

\section{CONCLUSIONES}


La RL puede convertirse en una referencia de alternativa para la conservación de la vegetación nativa dentro de propiedades rurales, inclusive recuperar los pasivos ambientales. Para esto la experiencia de Brasil es clave. Aun cuando la RL ha sido bastante atacada por productores rurales y desatendida por el Estado, representa una potencial herramienta para la conservación, especialmente en la Amazonia, con los avances del agronegocio y de otras actividades económicas que provocan la deforestación. Para esto la RL debe demostrar que es productiva desde el punto de vista sustentable y que sirve de apoyo para demás actividades productivas. Los productores rurales deben entender (como ya algunos lo han hecho) que la imagen creada alrededor de la RL es positiva y que ayuda a posicionar mejor sus productos en mercados internacionales.

\section{AGRADECIMIENTO}

El presente trabajo fue realizado con el apoyo parcial de la Coordenação de Aperfeiçoamento de Pessoal de Nível Superior - Brasil (CAPES) - Código de Financiamento 001 - para el primer autor.

\section{REFERENCIAS}

Ahrens, Sergio. «Sobre a Reserva Legal: origens históricas e fundamentos técnicoconceituais», Meio Ambiente e Acesso à Justiça. São Paulo, 2007. 691-707. http://www.planetaverde.org/arquivos/biblioteca/ arquivo_20140212142900_2726.pdf.

Azevedo, Andrea A., Raoni Rajão, Marcelo A. Costa, Marcelo C. C. Stabile, Marcia N. Macedo, Tiago N. P. dos Reis, Ane Alencar, Britaldo S. Soares-Filho, y Rayane Pacheco. «Limits of Brazil's Forest Code as a Means to End Illegal Deforestation». Proceedings of the National Academy of Sciences 114, Vol29 (18 de julio de 2017): 7653-7658. doi:10.1073/pnas.1604768114. 
Azevedo, Andrea A., Marcelo C. C. Stabile, y Tiago N. P. Reis. «Commodity Production in Brazil: Combining Zero Deforestation and Zero Illegality». Elementa: Science of the Anthropocene 3 (2 de diciembre de 2015): 1-12. doi:10.12952/journal.elementa.000076.

Castro, Daniel Stella. «A instituição da Reserva Legal no Código Florestal brasileiro: fundamentos histórico-conceituais». Geography Department, University of Sao Paulo, 2013: 132-54. doi:10.7154/RDG.2013.0026.0007.

CEPEA. «PIB do agronegócio brasileiro», 2019. www.cepea.esalq.usp.br/br/pib-doagronegocio-brasileiro.aspx.

Coalizão Brasil. «Em defesa do código florestal», 2019. http://www.coalizaobr.com.br/home/emdefesadocodigoflorestal.

Conservation International, y World Wildlife Fund. «PADDDtracker.org Data Release Version 2.0 (May 2019). Arlington, VA: Conservation International. Washington, DC: World Wildlife Fund.», 2019.

Duffy, Philip B., Paulo Brando, Gregory P. Asner, y Christopher B. Field. «Projections of Future Meteorological Drought and Wet Periods in the Amazon». Proceedings of the National Academy of Sciences 112, Vol43 (27 de octubre de 2015): 13172-77. doi:10.1073/pnas.1421010112.

Kengen, Sebastião. «A política florestal Brasileira: uma perspectiva histórica». Porto Seguro, Brasil: Instituto de Pesquisas e estudos Florestais, 2001.

Lei $\mathrm{n}^{\circ}$ 4.771/1965, de 15 de septiembre de 1965. Código Florestal. Presidência da República. http://www.planalto.gov.br/ccivil_03/leis/14771.htm.

Lei no 12.651/2012, de 25 de mayo de 2012. Código Florestal. Presidência da República. http://www.planalto.gov.br/ccivil_03/_ato2011-2014/2012/lei/112651.htm.

Mascia, Michael B., y Sharon Pailler. «Protected Area Downgrading, Downsizing, and Degazettement (PADDD) and Its Conservation Implications: PADDD and Its Implications». Conservation Letters 4, Vol1 (febrero de 2011): 9-20. doi:10.1111/j.1755263X.2010.00147.x.

Medeiros, Rodrigo, y Marta Irving. «A proteção da natureza no Brasil: evolução e conflitos de um modelo em construção». RDE-Revista de Desenvolvimento econômico 6, Vol9 (2004): 11.

Metzger, Jean P. «Bases biológicas para Reserva Legal». Ciência Hoje 31, Vol 183 (junio de 2002): 48-49.

Moutinho, Paulo, IPAM, IMAZON, GREENPEACE, IMAFLORA, ICV, ISA, TNC, y WWF. «A Pathway to Zero Deforestation in the Brazilian Amazon Full Report», 2017, 33. 
Rajão, Raoni, Vinícius Faria, Tomás Carvalho, Felipe Cerignoni, Isabella Granero, Luís Fernando Guedes Pinto, Vivian Ribeiro, et al. «A quem interessa a MP 867 do Código Florestal?» Observatorio do Código Florestal, 2019.

Rivera, Claudio, y Arturo Vallejos-Romero. «La privatización de la conservación en Chile: repensando la gobernanza ambiental». Bosque (Valdivia) 36, Vol 1 (2015): 15-25. doi:10.4067/S0717-92002015000100003.

Santiago, Thaís Muniz Ottoni, José Luiz Pereira de Rezende, y Luís Antônio Coimbra Borges. «A evolução da reserva legal: fundamentos para análise e compreensão do instrumento». Ciência Rural 47, Vol 2 (2017): 237-264. doi:10.1590/0103$8478 \mathrm{cr} 20141349$.

SFB. «Cadastro Ambiental Rural Boletim Informativo». Datos hasta 31 de julio de 2019, 2019. http://www.florestal.gov.br/documentos/car/boletim-do-car/4211-boletiminformativo-julho-de-2019/file.

Silva, Benedito, Claudia Azevedo-Ramos, y Hilder Farias. «The impact of decentralization policies: the environmental performance applied to mucipalities of the Amazon». Desenvolvimento e meio ambiente 51 (agosto de 2019): 202-223.

Sociedade Brasileira para o Progresso da Ciência, y Academia Brasileira de Ciências. O Código Florestal e a Ciência: contribuições para o diálogo. SBPC., 2012. http://www.sbpcacervodigital.org.br/bitstream/20.500.11832/2634/2/Livro\%20Co digoFlorestal__2aed.pdf.

Stabile, Marcelo C. C., André L. Guimarães, Daniel S. Silva, Vivian Ribeiro, Marcia N. Macedo, Michael T. Coe, Erika Pinto, Paulo Moutinho, y Ane Alencar. «Solving Brazil's Land Use Puzzle: Increasing Production and Slowing Amazon Deforestation». Land Use Policy, 19 de noviembre de 2019, 104362. doi:10.1016/j.landusepol.2019.104362.

UNEP-WCMC. «Protected Area Profile for Latin America \& Caribbean from the World Database of Protected Areas, September 2019», 2019. www.protectedplanet.net.

UNEP-WCMC, y IUCN. Protected Planet Report 2016. UNEP-WCMC and IUCN: Cambridge UK and Gland, Switzerland., 2016.

Pádua, José Augusto. "Os Fundamentos Históricos Da Conservação Florestal No Brasil." In Ensaios Em Ciências Ambientais: Crises, Riscos e Racionalidades, editado por Sandro Dutra e Silva, 185-216. Rio de Janeiro: Garamond, 2016.

Soares-Filho, Britaldo, Raoni Rajão, Marcia Macedo, Arnaldo Carneiro, William Costa, Michael Coe, Hermann Rodrigues, and Ane Alencar. "Cracking Brazil's Forest Code." Science 344, no. 6182 (Abril 25, 2014): 363-64. doi:10.1126/science.1246663.

Sparovek, Gerd, Göran Berndes, Alberto Giaroli de Oliveira Pereira Barretto, and Israel Leoname Fröhlich Klug. "The Revision of the Brazilian Forest Act: Increased Deforestation or a Historic Step towards Balancing Agricultural Development and 
Nature Conservation?" Environmental Science \& Policy 16 (Febrero 2012): 65-72. doi:10.1016/j.envsci.2011.10.008.

\title{
The experience of the Legal Reserve in Brazil: a conservation option in rural properties.
}

\begin{abstract}
The Legal Reserve (LR) is a protection tool oriented to conserve part of the natural resources within rural properties in Brazil. For almost a century, it has been the centre of debates between farmers and environmentalists. Through a literature review, this study explains the evolution of the LR highlighting its contributions, even for agriculture. Currently, part of farmers supports the LR in response to markets that demand more sustainable behavior. With $100 \%$ of properties with registered LR and $80 \%$ compliance with regulations, the LR begins to fulfill its function. A better understanding of this instrument allows other countries to assess its potential use as a conservation tool.
\end{abstract}

Keywords: Brazil, landscape planning, environmental policy, sustainability.

Recibido: 10/01/2020

Aprobado: 02/04/2020 